Opposed Port Alignment System (OPAS): A Commercial Astronomical Telescope Modified for Viewing the Interior of the NIF Target Chamber

A. M. Manuel, T. J. McCarville, L. G. Seppala, J. L. Klingmann, D. H. Kalantar

August 10, 2012

Opposed Port Alignment System (OPAS): A Commercial Astronomical Telescope Modified for Viewing the Interior of the NIF Target Chamber

San Diego, CA, United States

August 12, 2012 through August 16, 2012 
This document was prepared as an account of work sponsored by an agency of the United States government. Neither the United States government nor Lawrence Livermore National Security, LLC, nor any of their employees makes any warranty, expressed or implied, or assumes any legal liability or responsibility for the accuracy, completeness, or usefulness of any information, apparatus, product, or process disclosed, or represents that its use would not infringe privately owned rights. Reference herein to any specific commercial product, process, or service by trade name, trademark, manufacturer, or otherwise does not necessarily constitute or imply its endorsement, recommendation, or favoring by the United States government or Lawrence Livermore National Security, LLC. The views and opinions of authors expressed herein do not necessarily state or reflect those of the United States government or Lawrence Livermore National Security, LLC, and shall not be used for advertising or product endorsement purposes. 


\title{
OPPOSED PORT ALIGNMENT SYSTEM (OPAS): A COMMERCIAL ASTRONOMICAL TELESCOPE MODIFIED FOR VIEWING THE INTERIOR OF THE NIF TARGET CHAMBER
}

\author{
Anastacia M. Manuel*, Tom J. McCarville, Lynn G. Seppala, Jeff L. Klingmann, Daniel H. Kalantar
} Lawrence Livermore National Laboratory, 7000 East Avenue, Livermore, CA, USA 94550

\begin{abstract}
The National Ignition Facility (NIF) requires high resolution live images of regions inside the target chamber in order to align diagnostic instruments to fusion targets and to monitor target stability. To view the interior of the target chamber, we modified a commercial 11-inch Schmidt-Cassegrain telescope to develop the Opposed Port Alignment System (OPAS). There are two OPAS systems installed on the target chamber ports directly opposite the diagnostics. This paper describes the optical design, highlighting the two key modifications of the telescope. The first key modification was to reposition the Schmidt corrector plate and to uniquely mount the secondary mirror to a precision translation stage to adjust focus from $5.5 \mathrm{~m}$ to infinity. The stage is carefully aligned to ensure that the telescope's optical axis lies on a straight line during focus adjustments. The second key modification was a custom three element lens that flattens the field, corrects residual aberrations of the Schmidt-Cassegrain and, with a commercial 1:1 relay lens, projects the final image plane onto a large format 50 mega-pixel camera. The OPAS modifications greatly extend the Schmidt-Cassegrain telescope's field of view, producing nearly diffraction-limited images over a flat field covering \pm 0.4 degrees. Also discussed in the paper are the alignment procedure and the hardware layout of the telescope.
\end{abstract}

Keywords: NIF, Telescope, Alignment

\section{INTRODUCTION}

The National Ignition Facility (NIF) is a high energy laser system at Lawrence Livermore National Laboratory, designed to study inertial confinement fusion and numerous other experiments. ${ }^{1}$ The 192 beams of the laser are directed at small targets located at the center of the 5-m radius spherical vacuum chamber. Diagnostics used to verify beam alignment in the target chamber and the target location are described in companion papers at this conference. ${ }^{2,3}$ Other diagnostics quantify the target performance through x-ray and neutron radiation measurements. The target emission diagnostics are inserted on a cart, which rides on rails into the target chamber, known as a Diagnostic Instrument Manipulator (DIM). ${ }^{4}$ The three DIMs on NIF can position a diagnostic to within 0.3 to 1.5 meters proximity to the target and tip/tilt it relative to the target. To align the diagnostic to the target, a telescope located on the opposite side of the target chamber from the DIM provides a high resolution view of the diagnostic near the target chamber center (TCC). The optical design of this alignment telescope, known as the Opposed Port Alignment System (OPAS) is discussed in this paper.

There are two Opposed Port Alignment Systems, located in air, about $7 \mathrm{~m}$ away from chamber center on the equator of the target chamber opposite to the two equatorial DIMs (Figure 1). (There is no OPAS for the third DIM at the polar location.) OPAS needs to view objects that can be located at a variety of positions, including near the vicinity of the target chamber center (about $7 \mathrm{~m}$ away), the DIM insertion port at the far opposite side of the chamber (about $13 \mathrm{~m}$ away) and in the neutron imaging annex (about $35 \mathrm{~m}$ away). Therefore, the telescope requires a means to adjust its focus along a straight line of sight.

*manuel2@1lnl.gov; phone 925-423-2531 


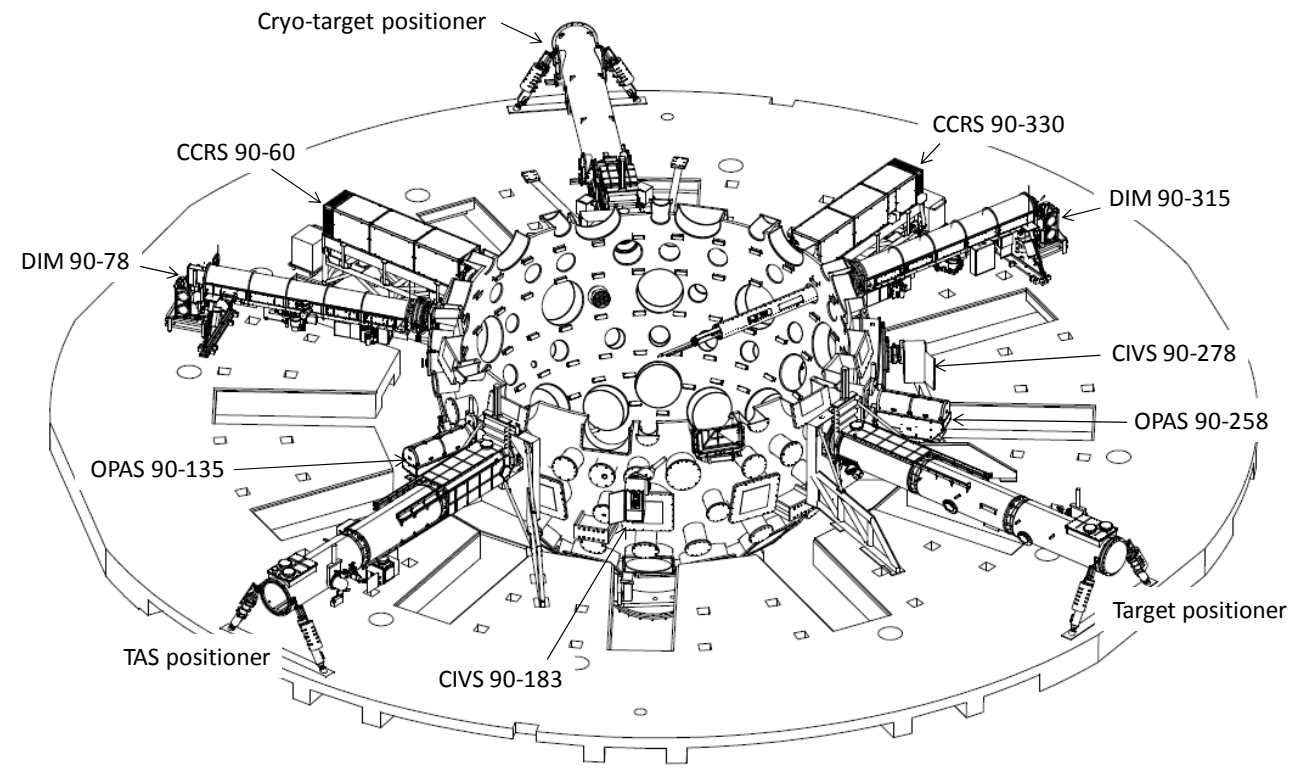

Figure 1. A section view of the target chamber. ${ }^{2}$ Each of the two Opposed Port Alignment System (OPAS) locations is on the opposite site of the chamber from a Diagnostic Instrument Manipulator (DIM).

During initial installation, the telescope's optical axis is aligned to a point at chamber center, and a second point centered on the DIM insertion port, and the optic axis then held fixed throughout its use. Because some target shots require diagnostics to be offset by up to $50 \mathrm{~mm}$ away from the OPAS optical axis, the telescope must have a field of view in excess of \pm 0.4 degrees. The radiation path within a diagnostic may require the diagnostic's aperture to be aligned accurate to within $100 \mu \mathrm{m}$ relative to the target, anywhere within this field of view. Of this total diagnostic alignment error, the fraction allocated to the OPAS telescope is only about $20 \mu \mathrm{m}$. Hence the OPAS telescope's alignment accuracy must be better than $\pm 1.5 \mu$ radians. The telescope resolution must be on this same order for accurate image processing. These requirements are summarized in Table 1 below.

Table 1. OPAS top level requirements.

\begin{tabular}{|lc|}
\hline \multicolumn{1}{c|}{ OPAS Performance Requirements } \\
Object distance & $5.5 \mathrm{~m}$ to $\infty$ \\
Field of view & \pm 0.4 degrees \\
Resolution at chamber center & $<20 \mu \mathrm{m}$ (Airy disc $\sim 25 \mu \mathrm{m})$ \\
Wavelength & $525 \pm 25 \mathrm{~nm}$ \\
Alignment accuracy & $\pm 1.5 \mu$ radians \\
\hline
\end{tabular}

\section{OPAS OPTICAL DESIGN}

OPAS is made of a commercial 11-inch Schmidt-Cassegrain telescope modified to meet the requirements for NIF. The base design of the telescope is described in Section 2.1 and the two modifications 1) to reposition the Schmidt corrector plate and to mount the secondary mirror on a precision translation stage and 2) to include a custom 3-element relay lens are described in Sections 2.2 and 2.3.

\subsection{Commercial telescope limitations}

The primary mirror, secondary mirror, and Schmidt corrector plate were extracted from a $279 \mathrm{~mm}$ diameter compact Schmidt-Cassegrain (SC) telescope manufactured by Celestron. ${ }^{5}$ In a compact SC design, the corrector plate is located near the focus of the spherical primary mirror (as opposed to near its center of curvature) which allows the optical tube length of the telescope to be much shorter, but increases the Petzval curvature. Using the system focal length of the commercial telescope of $f^{\prime}=2800 \mathrm{~mm}$ and Wilson's compact SC example ${ }^{6}$, we would expect the radius of curvature of the optimum image surface to be approximately

$$
r_{c(o p t)}=-\frac{f^{\prime}}{10.7}=\frac{2800 \mathrm{~mm}}{10.7}=261.7 \mathrm{~mm}
$$


Wilson suggests that this curvature is acceptable for visual use, but for photography, a field flattening lens is needed.

The commercial telescope optical design was reverse engineered using the element spacings, diameters and system f/\# provided by the manufacturer. Figure 2 shows the telescope focused for an object at target chamber center (TCC) by adjusting the position of the secondary mirror (as described in the following section). Using the Strehl ratio $>0.8$ rule of thumb, the maximum diffraction-limited field of view was found to be $\pm 10 \mathrm{~mm}$ ( \pm 0.08 degrees) at the (flat) telescope image plane, which is only $20 \%$ of our desired field of view.

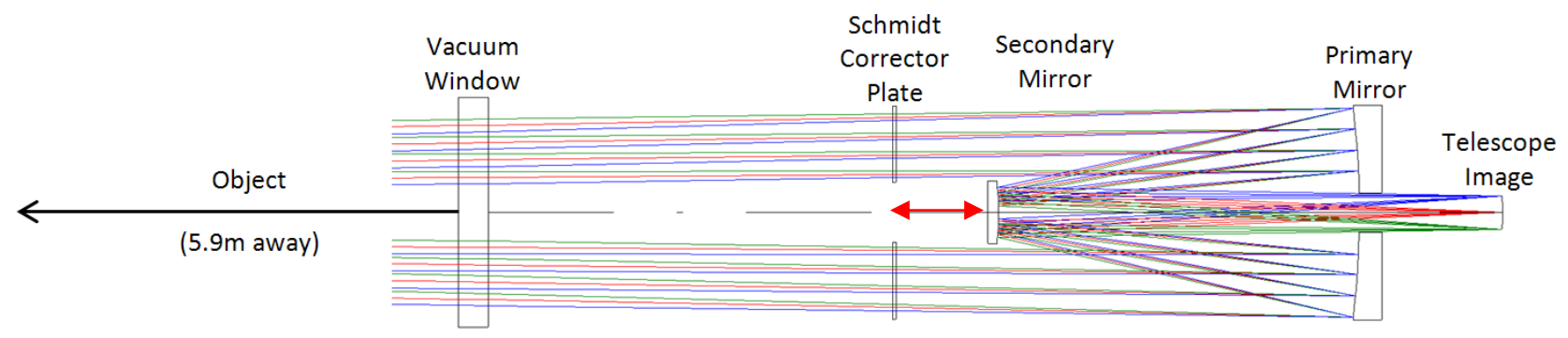

Figure 2. The optical layout of the OPAS telescope from the vacuum window to the telescope focus showing the on-axis field in red and $\pm 50 \mathrm{~mm}$ object size at TCC in blue and green. The radius of curvature of the telescope image plane showing is $228 \mathrm{~mm}$. The Schmidt corrector plate is moved $130 \mathrm{~mm}$ from its original position to correct spherical aberration through the focal range of infinity to $5.5 \mathrm{~m}$.

\subsection{Adjustable focus through secondary mirror motion}

In many optical systems, when the object distance changes, the camera position is just moved to the best focus. For our application, the object distance may range from the target chamber center to $28 \mathrm{~m}$ beyond it. The image plane shift $\left(\Delta z^{\prime}\right)$ scales with the product of object plane shift $(z)$ and local magnification at each object location $\left(m_{1}\right.$ and $\left.m_{2}\right)$ as shown in Equation 2:

$$
\Delta z^{\prime}=m_{1} m_{2} \Delta z=(0.445)(0.037)(28 \mathrm{~m})=0.46 \mathrm{~m} .
$$

It is impractical to maintain camera alignment on such a long translation stage. The easier focusing method is to adjust the distance between the primary and secondary mirrors. Since the secondary mirror is the smallest, it is the easier one to move. By adjusting the secondary mirror, the image plane stays at the same location and allows the corrective relay lenses (described in the following section) and the camera to remain stationary. In order for the telescope to image objects from the center of the target chamber to infinity, the secondary mirror only needs to move by $47 \mathrm{~mm}$.

For accurate alignment, the secondary mirror motion should be pure piston along the optic axis. Undesired secondary motion in other degrees of freedom (two transverse displacements and two rotations) would cause errors in the alignment of the object in the target chamber. The secondary mirror is fixed to the sliding platform of a motorized linear stage. The alignment error introduced by rotation of the stage platform when changing focus is reduced to an insignificant level by locating the secondary mirror's center of curvature at the moving platform. The stage is located along the telescope axis, and its profile fits within the area obscured by the secondary mirror. The stage/secondary assembly is supported by a thin truss passing through the primary mirror's field of view to minimize additional obscuration.

The original location of the Schmidt corrector plate in the Celestron design is coincident with the secondary mirror. As the telescope is refocused to points as close as $5.5 \mathrm{~m}$ (by increasing the distance between the primary mirror and the secondary mirror/corrector plate), 1.1 waves PV of over-corrected spherical aberration is generated. By repositioning the corrector plate $140 \mathrm{~mm}$ away from the secondary mirror, the spherical aberration can be corrected to less than 0.16 waves PV throughout the entire focal range. In effect, the beam diameter at the corrector plate and therefore the contribution of over-corrected spherical aberration are reduced. It is a fortunate coincidence that a solution with a reasonable spacing exists.

\subsubsection{Secondary mirror sensitivity}

Transverse displacement error of the secondary mirror stage platform results in a pointing shift (object placement error) equal to 


$$
x=\frac{2 L \Delta}{R m_{S C}}=\frac{2(0.67 \mathrm{~m}) \Delta}{(0.33 \mathrm{~m})(0.445)}=9 \Delta
$$

where $L$ is the distance from the secondary to the intermediate image plane, $R$ is the secondary mirror radius of curvature, $m_{S C}$ is the SC telescope magnification, and $\Delta$ is the transverse displacement of the secondary mirror. Thus, object placement error is about 9 microns per micron of transverse error in the secondary. The secondary mirror's motion along the optic axis is less than $20 \mathrm{~mm}$ over the working distance for diagnostic alignment (within the target chamber), and it was not difficult to find a stage that would limit object placement error from this contribution to \pm 10 microns.

\subsection{Custom relay lens design}

The second telescope modification was necessary to meet the large field of view required. In this modification, just three small custom lenses, plus an off-the-shelf 1:1 relay lens are used to flatten the field and correct the residual aberrations of the Schmidt-Cassegrain telescope. The relay lens design provides near diffraction-limited performance, minimal distortion, and resizes the telescope image to fit the camera format. To achieve diffraction-limited performance, the three lenses were designed to correct the field curvature and third order astigmatism of the Schmidt-Cassegrain telescope without adding additional coma or spherical aberration (which are already corrected in the base telescope design). Two different glasses were used to minimize axial and lateral color over the operational wavelength range (500-550nm). (The SC has extremely little chromatic aberration by itself because the only refractive component is the Schmidt corrector plate which has nearly zero power.) The three lenses project the real image of the telescope behind the primary mirror onto a virtual image which is de-magnified and not too far away from the original telescope image plane. In this way, these lenses act similarly to a Bravais lens. ${ }^{7,8}$ The virtual image of the 3 -lens relay is projected $1: 1$ by the off-the-shelf 6-element imaging lens (Apo-Rodagon-D, ${ }^{9} 75 \mathrm{~mm}$ effective focal length f/4) onto the camera (Figure 3). The Apo-Rodagon-D lens was modeled in the optical software program as a lens module which adds no aberration because the lens prescription was not available; however this is a reasonable approximation for a well-designed commercial lens.

A large format camera is needed both to capture the wide field of view and to preserve the resolution. For example, with a $100 \mathrm{~mm}$ diameter field and a minimum 20 microns per pixel (in object space), 5000 pixels across are required. The $50 \mathrm{MP}$ camera, manufactured by illunis LLC, ${ }^{10}$ has CCD chip size of $49.1 \mathrm{~mm} \times 36.8 \mathrm{~mm}$ with $6 \mu \mathrm{m}$ pixels $(8176 \times 6132$ pixels $)$.

The system performance is nearly diffraction-limited over the entire field of view. For a 50 mm object radius at the target chamber center ( 0.4 degree field angle), the Strehl ratio is 0.78 and there is nearly no field curvature; the radius of curvature of the optimum image surface is $2.5 \mathrm{~m}$.

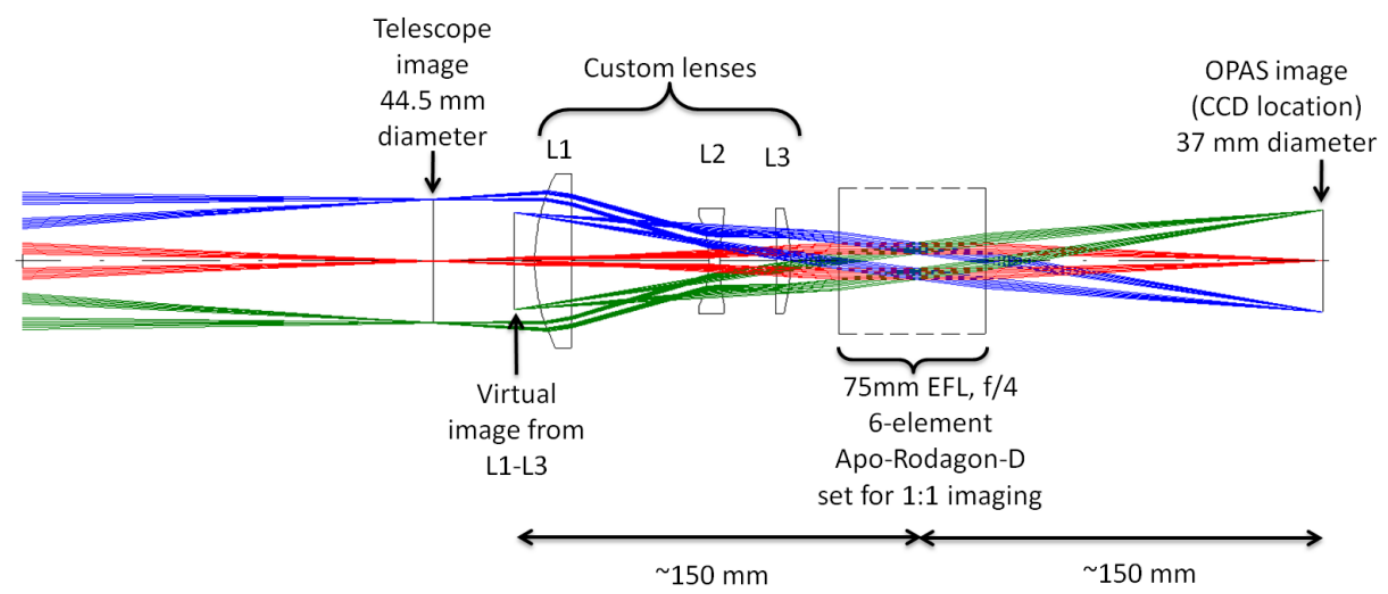

Figure 3. The optical layout of the OPAS telescope from the telescope focus to the final image plane showing the on-axis field in red and $\pm 50 \mathrm{~mm}$ object size at TCC $( \pm 0.4$ degrees $)$ in blue and green. 


\section{ALIGNMENT AND INSTALLATION}

The OPAS assembly was aligned on its $200 \mathrm{~mm}$ thick optical table in an off-line laboratory, as described in Sections 3.13.5. The tools used to align and assemble OPAS include a commercial alignment telescope modified to increase magnification and capture images on a CCD, and a bare $0.4 \mathrm{~mm}$ diameter multi-mode fiber, with LED fiber illumination. Once alignment was complete, the system was installed onto NIF, as described in Section 3.6.

\subsection{Primary mirror alignment}

Figure 4 illustrates the alignment axis used to align OPAS optics. The alignment axis was established by locating the fiber $19 \mathrm{~m}$ away from the alignment scope, and centering the fiber on the alignment scope's internal cross hair. The alignment magnification of the fiber image projected onto the scope CCD was approximately 0.1 .

The OPAS optical table was then placed in front of the alignment scope, and the primary mirror's inner aperture was centered within $\pm 0.5 \mathrm{~mm}$ of the alignment axis. The primary mirror pointing was set by moving a small scatter plate along the alignment axis, while viewing the scatter plate with alignment scope until the fiber image was in focus. The primary was then tipped and tilted until the fiber image was on the alignment axis. Averaging images over multiple frames removed the jitter from air turbulence over the long path.

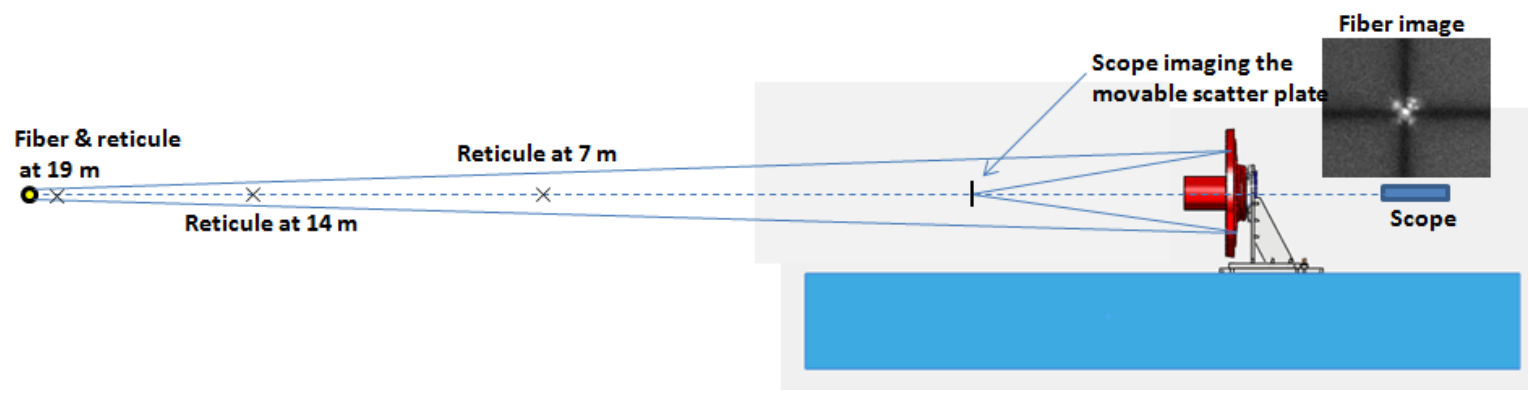

Figure 4. The primary mirror is aligned to the axis defined by the alignment telescope and the fiber source $19 \mathrm{~m}$ away. The Schmidt corrector plate, not shown in this illustration, was installed to its nominal location before the primary was aligned. The three transmission reticules on the alignment scope axis were used to verify the final alignment of the optics at different object distances.

\subsection{Secondary mirror alignment}

To align the secondary mirror over its entire range of motion, both the secondary mirror itself and the stage that it travels on need to be aligned. The stage axis is correctly aligned when the secondary mirror's center of curvature remains on the alignment axis for all OPAS working distances. First the secondary mirror stage axis was aligned roughly parallel to the alignment axis by placing a target on the stage's moving platform, moving the platform over its travel length, and observing the target's transverse motion with the alignment scope. The stage was tip/tilted until there was no observable transverse target displacement over the travel length.

The secondary mirror was then installed onto the stage to form an image of the fiber in back the primary mirror, as shown in Figure 5. The image location was identified by moving a transmission scatter plate behind the secondary along the alignment axis, while observing the scatter plate with the alignment scope. The secondary mirror was then tipped and tilted until the fiber image was centered on the alignment scope internal cross hair. This places the secondary mirror's center of curvature on the alignment axis at a fixed stage location, providing a starting point for iterative tip/tilt adjustment of the stage axis and secondary. 


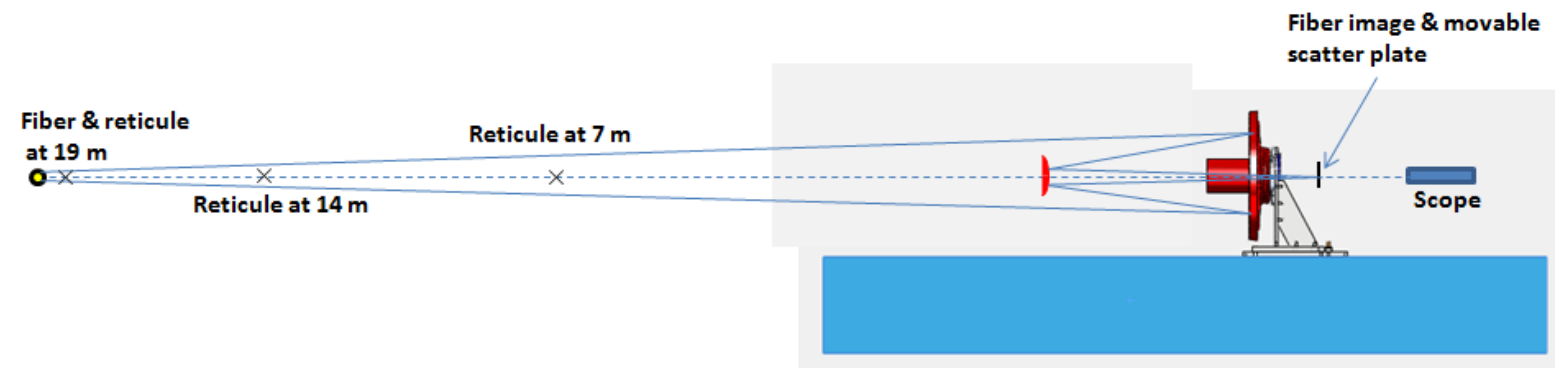

Figure 5. The fiber's image behind the primary mirror is used to align the secondary mirror. Moving the secondary mirror stage and following the image with the alignment scope allows precise alignment of both the stage axis and secondary mirror pointing.

Because the stage axis is not yet precisely parallel to the scope alignment axis, moving the secondary to a different object distance displaces its center of curvature from the alignment axis. As this occurs, the fiber image displacement from the alignment axis can be observed by moving the scatter plate to keep the fiber's image in focus, and observing the image with the alignment scope (Figure 6). The alignment scope is being used at close working distance with small focus adjustment, which limits the transfer of alignment scope internal errors to OPAS optics alignment.
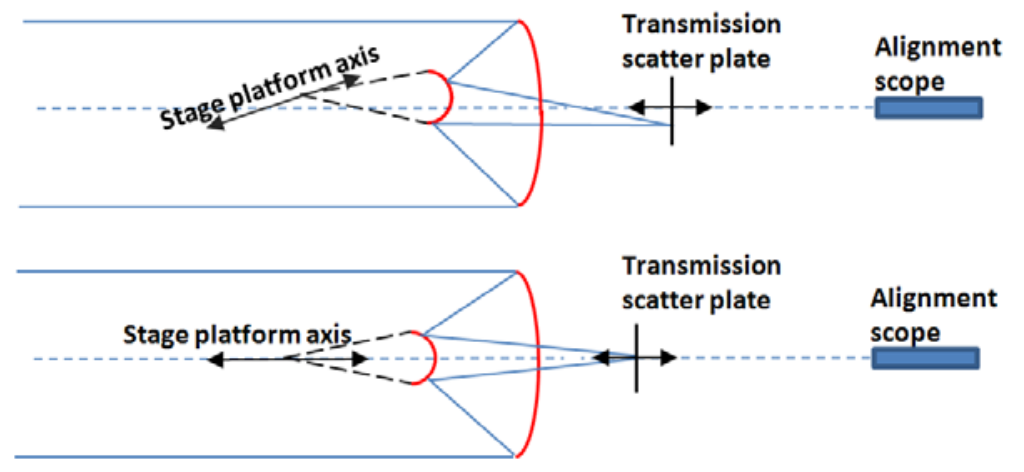

Figure 6. Top: The focus stage axis and the secondary's center of curvature are not aligned to the alignment axis.

Bottom: The fiber image remains on the alignment scope axis when the stage and secondary are properly aligned.

Observing the fiber image at two different stage axis positions (i.e., OPAS object distances) allows both stage axis and secondary pointing errors to be corrected by iteration. Figure 7 illustrates the location of pitch and yaw adjusters, as well as rotation axes, for the stage and secondary mirror. The alignment iteration begins by moving the secondary mirror to a far OPAS object distance, then tipping and tilting the secondary mirror until the fiber image returns to the alignment axis. The secondary mirror stage is then moved to a close OPAS object distance, and the stage axis is tipped and tilted until the fiber image returns to the alignment scope axis. This iteration "walks" the fiber image onto the alignment scope axis, within a pixel, for the entire range of the stages travel. Commercially available 100 pitch adjusters were used to drive pitch and yaw, and flexures designed into the assembly create the pitch and yaw axes. The secondary mirror is supported from the stage platform by the type of kinematic, spring loaded tip/tilt adjustment found on commercial mirror mounts. 


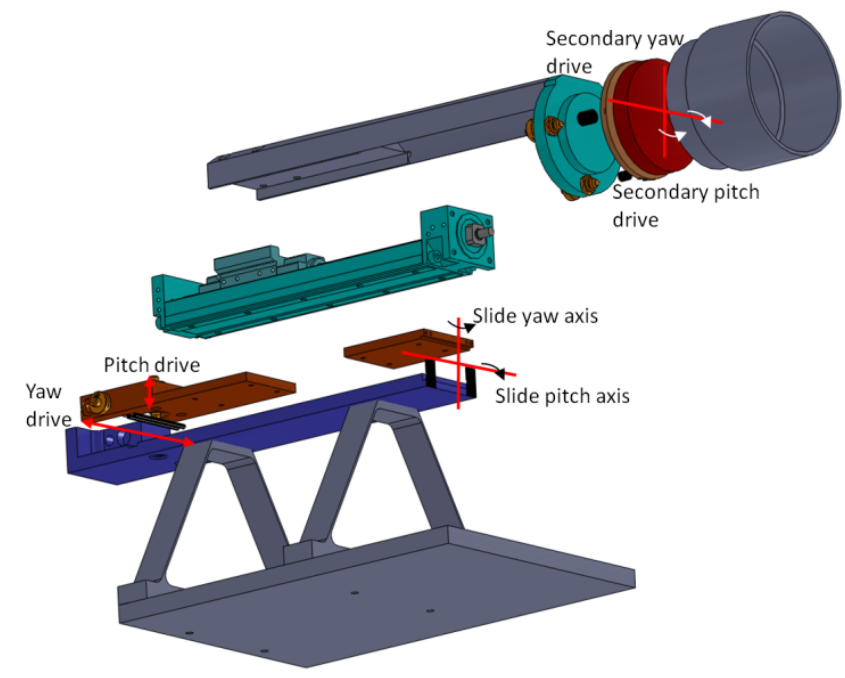

Figure 7. An exploded view of the secondary mirror assembly, illustrating the stage and mirror alignment features. The linear stage stepper motor and harmonic drive gear reducer are not shown.

\subsection{Primary and secondary mirror alignment verification}

Before the secondary mirror was installed, reticules were aligned along the alignment scope axis at 19,14 , and $7 \mathrm{~m}$ object distance. Viewing these reticules through OPAS, after the primary and secondary are adjusted, verifies that the mirrors are aligned to the alignment scope axis. To view the reticule images, the alignment scope is set to view the SC telescope focus (the intermediate image plane of OPAS), and the secondary mirror was translated to view the three reticules at $19 \mathrm{~m}, 14 \mathrm{~m}$, and $7 \mathrm{~m}$ sequentially. If the primary, secondary, and stage axis are properly aligned, the images should all appear centered on the alignment scope internal cross hair. The results are shown in Figure 8. The horizontal and vertical black lines are the internal cross hair of the alignment telescope. The two rotated lines are the 415 micron diameter wire cross hairs of the reticules. The image displacement is quite small for the three distances, indicating OPAS optics are aligned to within a few micro-radians.
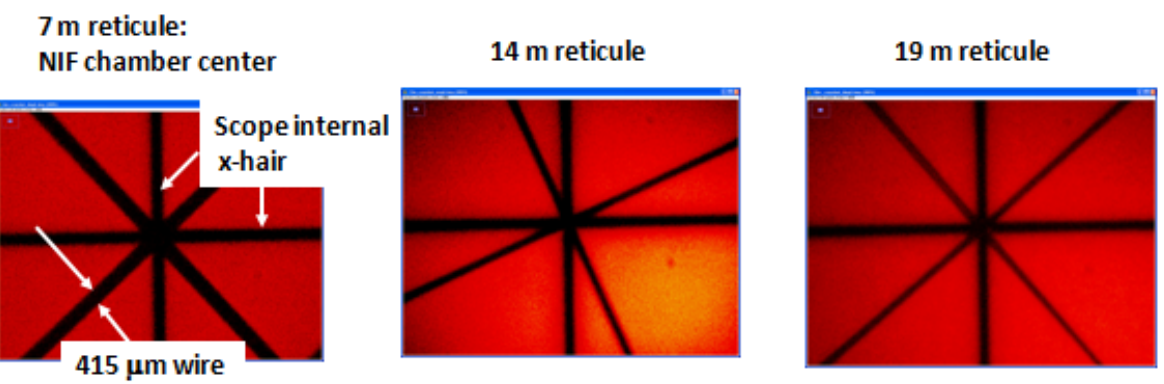

Figure 8. Alignment of wire reticules located at $19 \mathrm{~m}, 14 \mathrm{~m}$, and $7 \mathrm{~m}$ distance from OPAS shows the primary, secondary, and focus stage axis are well aligned. The horizontal and vertical lines are the alignment scope's internal cross hair, and the rotated lines are the wire reticule. The change in magnification for the different OPAS object distances is evident through the different thicknesses of the reticule wire.

\subsection{Schmidt corrector plate alignment}

Once OPAS's reflective optics were aligned, object resolution was checked by placing a line pattern resolution target at $7 \mathrm{~m}$ object distance, and observing the image with a CCD located at the intermediate image plane of OPAS. The observed resolution was significantly affected by small transverse displacements of the Schmidt corrector plate, and relatively insensitive to corrector plate displacement along the optic axis. This characteristic was used to optimize the Schmidt corrector plate's transverse location. The optimum resolution was about 30 microns per line pair, with $>20 \%$ contrast. This completed the adjustments to OPAS telescope optics. 


\subsection{Relay optics alignment}

The three corrective lenses and commercial imaging lens were centered in a custom barrel with spacers to set the distances between each lens precisely. This barrel and the camera were each mounted to a carriage that rides on a rail placed on the optical table. Prior to installing the relay/correction optics and CCD, a transmission reticule was placed on the optical table at the intermediate image plane, as shown in Figure 9. The edges of this reticule, visible in all OPAS images, serve as an alignment aid if the lenses and CCD removed and re-installed during use. The spacing between the intermediate image plane, lens barrel and CCD was adjusted by moving the carriage locations along the rail until the correct magnification was achieved for a $100 \mathrm{~mm}$ scale located $7 \mathrm{~m}$ away from OPAS. Finally, image resolution was checked to ensure there was no degradation over the field of view.

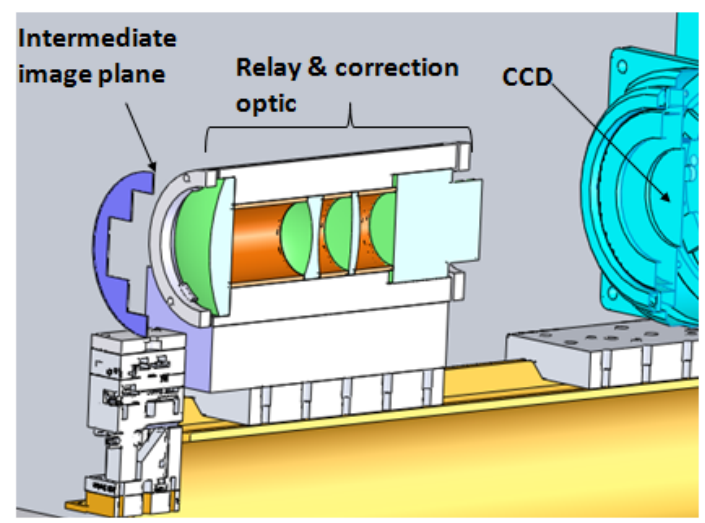

Figure 9. Cut away view of the intermediate image plane, relay/correction optics assembly and the illunis large format CCD that are located in back of the OPAS primary mirror.

\subsection{OPAS support structure and installation in target bay}

Once the off-line alignment of OPAS was complete, the system was installed in the NIF target bay. The optical table is supported by six adjustable length struts (Figure 10). The strut lengths are adjusted until the optical axis of OPAS is aligned with the center of a ball at target chamber center and with a cross hair mounted midway between the DIM rails at the location of the positioner gimbal.

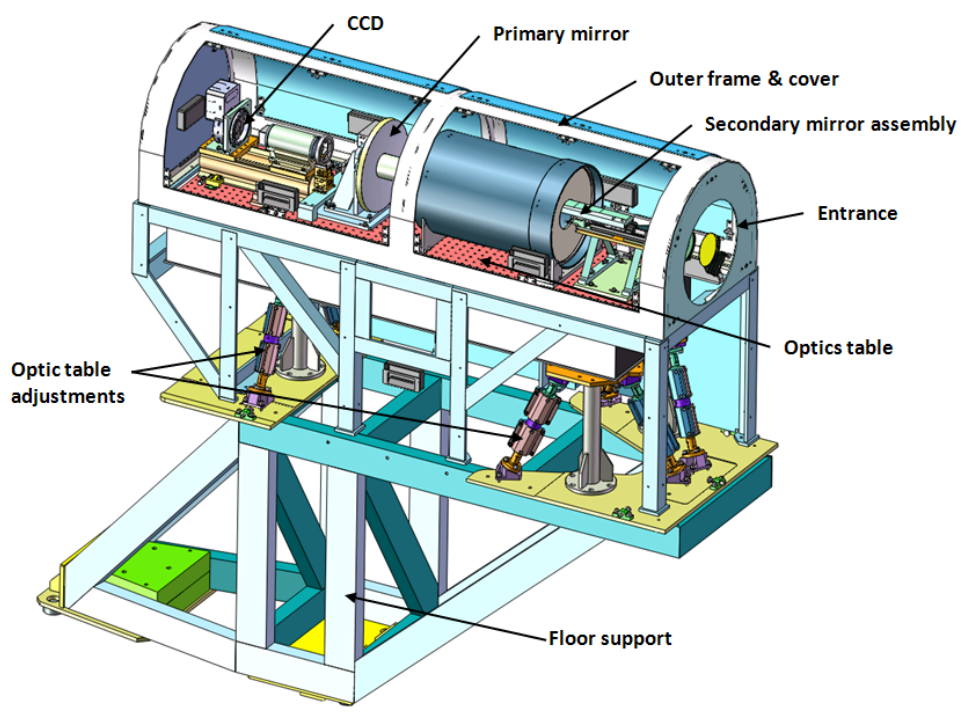

Figure 10. OPAS with cover plates removed for visibility into its interior.

The optical table and struts are surrounded by a sheet metal enclosure to limit stray light from the target bay onto the telescope images. All the above is supported off the concrete floor by a rigid, welded structure. 


\section{SUMMARY}

The Opposed Port Alignment System, used on NIF, is a based on a commercially available Schmidt-Cassegrain telescope. Modifications to the original system improved the diffraction-limited field of view by nearly a factor a five and allow the telescope to be used for a range of object positions. The only custom optics are three small lenses, which enabled us to spend our money where it counts, on a high-resolution camera. OPAS telescopes have been used to align diagnostics to NIF targets for over two years now and the quality of diagnostics data indicates OPAS meets its requirements.

We are evaluating improvements for stability. The target bay air around OPAS is controlled to a few tenths of a degree Celsius to limit thermally induced pointing drifts. Temperature fluctuations of a few tenths of a degree Celsius inside the OPAS enclosure are caused by power cycling of the camera, which is turned off and/or removed before some shots to prevent damage from neutrons. Precision levels indicate this temperature change induces an optical table pointing drift up to $10 \mu$ radians. Diagnostics will be misaligned if this drift occurs during the diagnostic alignment cycle. In addition, a new goal for OPAS is to monitor target stability right up to the time of a shot. This requires OPAS pointing to be stable within a micro-radian for a duration of a few hours. Methods of stabilizing the internal air temperature are being reviewed in preparation for this new requirement. The optimum means of stabilizing OPAS internal temperature will be identified within the coming year, in order to demonstrate pointing stability on the scale of a micro-radian.

Lawrence Livermore National Laboratory is operated by Lawrence Livermore National Security, LLC, for the U.S. Department of Energy, National Nuclear Security Administration under Contract DE-AC52-07NA27344.

\section{REFERENCES}

[1] Moses, E., and Wuest, C., "The National Ignition Facility: Status and Plans for Laser Fusion and High-EnergyDensity Experimental Studies," Fusion Science and Technology 43, 420-427 (2003).

[2] Kalantar, D.H., Di Nicola, P., Wood, R., Shingleton, N., McCarville, T., Klingmann, J., Dzenitis, J., Manuel, A.M., and Burkhart, S., "An overview of target and diagnostic alignment at the National Ignition Facility," Proc. SPIE $8505,(2012)$.

[3] Di Nicola, P., Kalantar, D. H., McCarville, T.J., et al., "Beam and target alignment at the National Ignition Facility using the Target Alignment Sensor (TAS)," Proc. SPIE 8505 (2012).

[4] Shingleton, N., Kalantar, D., Wood, R., McCarville, T., Klingmann, J., and Manuel, A., "Alignment of an X-ray imager line of sight in the National Ignition Facility (NIF) target chamber using a Diagnostic Instrument Manipulator (DIM) and Opposed-Port Alignment System (OPAS)," Proc. SPIE 8505, (2012).

[5] Celestron, "Celestron C11-A XLT (CGE) Optical Tube Assembly," <http://www.celestron.com/astronomy/opticaltubes/optical-tube-assemblies/celestron-c11-a-xlt-cge.html> (6 August 2012).

[6] Wilson, R.N., [Reflecting Telescope Optics I], Second Edition, Springer, 186-191 (2007).

[7] Smith, W.J., [Modern Optical Engineering], Third Edition, McGraw-Hill, 289 (2000).

[8] Griffith, J.D., "Bravais lens," Patent \#5966252, Rochester, NY, (1999).

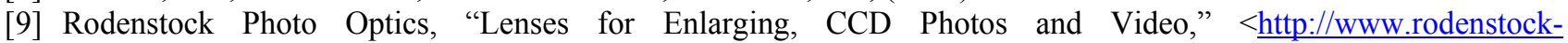
photo.com/mediabase/original/e_Rodenstock_Printing_CCD_43-62_8230.pdf $>$ (6 August 2012).

[10] Illunis, "RMV 50M," <http://www.illunis.com/products/FullFrame/50MP/50MP.html> (6 August 2012). 